\title{
Twist mediates an aggressive phenotype in human colorectal cancer cells
}

\author{
JUN-JIAN DENG ${ }^{1}$, WEI ZHANG ${ }^{2}$, XI-MING XU ${ }^{1}$, FAN ZHANG $^{3}$, \\ WEI-PING TAO ${ }^{1}$, JUN-JIE YE ${ }^{1}$ and WEI GE ${ }^{1}$
}

Departments of ${ }^{1}$ Cancer Center, ${ }^{2}$ Obstetrics and Gynecology, ${ }^{3}$ Pharmacy, Renmin Hospital of Wuhan University, Wuhan, Hubei 430060, P.R. China

Received October 18, 2015; Accepted December 8, 2015

DOI: $10.3892 /$ ijo.2016.3342

\begin{abstract}
Epithelial-mesenchymal transition (EMT) is a crucial process providing cancer cells with the ability to migrate and metastasize to distant sites. Recently, EMT was shown to be associated with the cancer stem cell (CSC) phenotype and chemoresistance. Twist is a transcription factor that regulates EMT in a various cancer cells, including colorectal cancer (CRC). Our study was done to determine the role of Twist in mediating aggressive phenotype in CRC. Human CRC cell lines were transduced with a retroviral Twist construct or vector control. Migration and invasion abilities were determined in vitro using modified Boyden chamber assays. Mammosphere formation assay was performed to detect CSC characteristics. EMT and CSC markers were detected using western blotting and RT-PCR. Chemosensitivity to oxaliplatin of the transfected cells were determined by the MTT assay. Human CRC specimens were stained for Twist and P-gp expression. Twist overexpression triggered EMT and a CSC-like phenotype in human CRC cells and enhanced cell migration, invasion and mammosphere formation abilities. In addition, Twist-overexpressing CRC cells were more chemoresistant to oxaliplatin than control cells. Furthermore, Twist overexpression increased P-gp expression in CRC cells, which is a transmembrane glycoprotein conferred multidrug-resistance phenotype to various cancer cells. Importantly, Twist and P-gp were expressed correlatively in human CRC specimens. Thus, Twist is a potential therapeutic target in metastatic CRC.
\end{abstract}

\section{Introduction}

Epithelial-mesenchymal transition (EMT), a crucial phenotypic conversion during embryonic development, wound healing and tissue remodeling, plays an essential role in

Correspondence to: Dr Wei Zhang, Department of Obstetrics and Gynecology, Renmin Hospital of Wuhan University, Wuhan, Hubei 430060, P.R. China

E-mail: 18949850180@163.com

Key words: Twist, epithelial-mesenchymal transition, cancer stem cell, chemoresistance, P-gp tumor invasion and metastasis (1-3). As a reversible process, EMT often occurs at the invasive front of many metastatic cancers (4). Different signals received from tumor microenvironment can trigger EMT conversion, such as TGF $\beta$, TNF $\alpha$, EGF and Notch $(5,6)$. During EMT conversion, epithelial cells lose intercellular adhesion, acquire mesenchymal characteristics and increase migratory and invasive capabilities (7). The hallmark of EMT is loss of E-cadherin expression (1). A set of transcription factors have been implicated in the regulation of EMT, including Twist, Snail, Slug and ZEB1 (8). Twist, a member of zinc-finger transcription factor, has been proved as a central EMT regulator. Studies showed that Twist induces EMT by binding to the E-box site in the promoter of E-cadherin and repressing E-cadherin transcription (9). Twist plays an important role in the pathogenesis of various malignant tumors, predominantly by promoting invasiveness and metastatic behavior. Twist is overexpressed in a variety of cancers, including breast cancer, hepatocellular carcinoma, prostate cancer, head and neck squamous cell carcinoma and ovarian cancer (9). In breast cancer and lung cancer, Twist expression is associated with a more aggressive phenotype and poorer clinical outcome (10). Furthermore, several studies revealed a correlation between Twist expression and metastatic disease. Twist expression levels were positively correlated with high grade and metastasis of prostate cancer and bladder cancer $(11,12)$.

Recently, increasing studies suggested that EMT phenotype also led to the acquisition of other properties involved in tumor progression, such as increased resistance to chemotherapy drug and acquisition of cancer stem cell (CSC)-like features (13). The CSCs are a group of cells within a tumor that possess the capacity to self-renew and differentiate into the heterogeneous lineages of cancer cells which compose the whole tumor (14). Mounting studies showed that somatic cells can be reprogramed into pluripotent stem-like cells via co-expression of pluripotent makers such as Bmi-1, Nanog and CD44 (15). A recent study confirmed that drug-resistant lung cancer cells display a cancer stem-like trait and EMT phenotype (16). Taken together, these observations suggested that EMT and CSC-like properties are mechanistically associated to chemoresistance.

Colorectal cancer (CRC) is a major worldwide health concern. Most deaths from CRC are due to metastases that are 
resistant to conventional therapies. Analysis of Twist in CRC specimens in a recent study suggested that Twist can be an important marker of poor prognosis (17). However, the specific role of Twist in aggressive phenotype of CRC is has not been fully elucidated. In this study we showed that upregulation of Twist confers aggressive phenotype of CRC cells by inducing EMT and CSC properties. Moreover, we demonstrated that high levels of Twist expression in CRC cells are linked to increased chemoresistance.

\section{Materials and methods}

Chemicals and reagents. Primary antibody against E-cadherin was obtained from Cell Signaling Technology (Danvers, MA, USA). Primary antibodies against $\mathrm{N}$-cadherin, vimentin, Twist, P-gp and $\alpha$-tubulin were obtained from Santa Cruz Biotechnology (Santa Cruz, CA, USA). Primary antibodies against Bmi-1 and Nanog were obtained from Epitomics. Alexa Fluor 488/594 conjugated secondary antibody, Horseradish peroxidase (HRP)-conjugated secondary antibody, DAPI and Lipofectamine 2000 were from Invitrogen (Carlsbad, CA, USA). PrimeScript ${ }^{\circledR}$ RT reagent kit and SYBR ${ }^{\circledR}$ Premix Ex Taq ${ }^{\mathrm{TM}}$ were from Takara. E.Z.N.A ${ }^{\circledR}$ HP Total RNA kit was obtained from Omega Bio-Tek (Doraville, GA, USA).

Cell culture. The colorectal carcinoma cell lines HCT116 and SW480 were obtained from the Type Culture Collection of the Chinese Academy of Sciences (Shanghai, China). SW480 cells were cultured in DMEM culture medium (Gibco BRL) supplemented with $10 \%$ fetal bovine serum, and HCT116 cells were maintained in McCoy'5a culture medium (Gibco BRL) supplemented with $10 \%$ fetal bovine serum under a humidified $5 \% \mathrm{CO}_{2}$ atmosphere at $37^{\circ} \mathrm{C}$ in an incubator.

Transwell migration and invasion assay. Invasion and migration assays were performed in Boyden chambers. The polycarbonate filters, pre-coated with Matrigel matrix, were used for invasion assay, and uncoated filters were used for migration assay. Cells $\left(1 \times 10^{5}\right)$ in $300 \mu 1$ medium (containing $0.1 \%$ FBS) transfected with empty vector plasmid or pcDNATwist were seeded in the upper chamber. Then $600 \mathrm{ml}$ medium with $10 \%$ FBS, served as a chemotactic agent, and was added to the lower chamber. For migration, after $24 \mathrm{~h}$ incubation, the cells migrated onto the lower chamber were fixed with $4 \%$ paraformaldehyde for $30 \mathrm{~min}$, stained with hematoxylin and counted under microscope. For invasion, the cells in the upper chamber were fixed in $4 \%$ paraformaldehyde for $30 \mathrm{~min}$. Then the Matrigel was removed from the filter with a cotton swab. The cells adhered to the underside of the filter were stained with hematoxylin and counted under a microscope. Each assay was repeated in three independent experiments.

Gene overexpression. Cells/well $\left(2 \times 10^{5}\right)$ were seeded on a 6 -well plate and left in culture until the next day. They were then transfected with $2 \mu \mathrm{g}$ empty vector plasmid or pcDNATwist mixed with Lipofectamine 2000 reagent in serum-free medium according to the manufacturer's instructions. After $6 \mathrm{~h}$, medium was changed to complete culture medium and the cells were incubated for another 24-48 $\mathrm{h}$ before harvest.
Quantitative real-time PCR. Total mRNA of the cells was extracted after transfected with plasmids for $24 \mathrm{~h}$. First step cDNA synthesis was generated from $500 \mathrm{ng}$ total mRNA. Quantification of target and reference genes were detected in triplicate on LightCycler ${ }^{\circledR} 480$ II. The primers used in each reaction are shown in Table I. Expression levels for each target gene were normalized to GAPDH gene. Results were calculated using the comparative threshold cycle $(\Delta \Delta \mathrm{CT})$ method. Data are presented as the mean \pm standard deviation (SD) from three independent experiments.

Western blot analysis. The cells were washed three times with ice-cold PBS and then lysed in lysis buffer. Lysates were cleared by centrifugation and denatured by boiling for 5 min in Laemmli buffer. Equal quantity of protein samples were loaded per well and separated on SDS-polyacrylamide gels, and then transferred electrophoretically onto PVDF membranes. After blocked with 5\% non-fat milk at room temperature for $2 \mathrm{~h}$, membranes were incubated with primary antibodies at $4^{\circ} \mathrm{C}$ overnight and then incubated with HRP-conjugated secondary antibodies for $1.5 \mathrm{~h}$ at room temperature. Immune complexes were detected using Western Blotting Plus chemiluminescence reagent.

Immunofluorescence. The cells transfected with plasmids were cultured on chamber slides for $24 \mathrm{~h}$, then washed three times with PBS, fixed with $4 \%$ paraformaldehyde for $30 \mathrm{~min}$ and permeabilized with $0.3 \%$ Triton X-100 for $10 \mathrm{~min}$. After blocked with goat serum for $2 \mathrm{~h}$ at room temperature, cells were incubated with antibodies against E-cadherin, vimentin (1:100 dilution) at $4^{\circ} \mathrm{C}$ overnight. Slides were washed three times with PBS and incubated with Alexa Fluor 488 or Alexa Fluor 594-conjugated secondary antibodies (1:1,000 dilution) for $1 \mathrm{~h}$ at room temperature. Nuclei were stained with DAPI $(10 \mu \mathrm{g} / \mathrm{ml})$ for $10 \mathrm{~min}$. Samples were examined with Confocal Laser Scanning Microscopy (Zeiss) to analyze expression of E-cadherin, vimentin.

Mammosphere formation assay. Stem cells are enriched in mammospheres of cancer cells and assay is based on the ability of stem cells to grow and form spheres in serum-free medium. Mammosphere culture was done in a serum-free DMEM/F12 supplemented with $20 \mathrm{ng} / \mathrm{ml} \mathrm{EGF,} \mathrm{B27,} 20 \mathrm{ng} / \mathrm{ml}$ basic fibroblast growth factor (bFGF), $1 \mathrm{~g} / \mathrm{ml}$ hydrocortisone, $5 \mathrm{~g} / \mathrm{ml}$ insulin and $1 \%$ antibiotic-antimycotic. Single cells prepared from mechanical dissociation were plated in 12-well ultralow attachment plates at a density of 1,000 cells $/ \mathrm{ml}$ in culture. Single cell status was confirmed under a microscope. Fresh mammosphere culture was added every 3-4 days. After 7 days of culture, the number of mammospheres $(>20 \mu \mathrm{m})$ was counted under an upright microscope and the images were acquired with the upright microscope. Each assay was carried out in triplicate and repeated in three independent experiments.

Cell viability assay. Cell viability was performed using the MTT assay. Briefly, cells $\left(1 \times 10^{4}\right)$ transfected with plasmids were seeded into each well of a 96-well plate and treated with oxaliplatin at concentrations from 5 to $20 \mu \mathrm{M}$ for $24 \mathrm{~h}$. After treatment, the cells were washed twice with PBS, and $100 \mathrm{ml}$ of $0.25 \mathrm{mg} / \mathrm{ml} \mathrm{MTT}$ inculture medium was added to 
Table I. Primers used in this study.

\begin{tabular}{|c|c|c|}
\hline Gene & Forward primer 5'-3' & Reverse primer $5^{\prime}-3^{\prime}$ \\
\hline Twist & GGAGTCCGCAGTCTTACGAG & TCTGGAGGACCTGGTAGAGG \\
\hline E-cadherin & TACACTGCCCAGGAGCCAGA & TGGCACCAGTGTCCGGATTA \\
\hline Vimentin & TGAGTACCGGAGACAGGTGCAG & TAGCAGCTTCAACGGCAAAGTTC \\
\hline Bmi-1 & TGGAGAAGGAATGGTCCACTTC & GTGAGGAAACTGTGGATGAGGA \\
\hline Nanog & CAAAGGCAAACAACCCACTT & TCTGCTGGAGGCTGAGGTAT \\
\hline CD44 & GACACATATTGTTTCAATGCTTCAGC & GATGCCAAGATGATCAGCCATTCTGGAAT \\
\hline $\mathrm{ABCB} 1$ & TGCTCAGACAGGATGTGAGTTG & AATTACAGCAAGCCTGGAACC \\
\hline $\mathrm{ABCC} 1$ & GCCAAGAAGGAGGAGACC & AGGAAGATGCTGAGGAAGG \\
\hline $\mathrm{ABCC} 2$ & TGGTGGCAACCTGAGCATAGG & ACTCGTTTTGGATGGTCGTCTG \\
\hline ABCC3 & CTTAAGACTTCCССТCAACATGC & GGTCAAGTTCCTCTTGGCTC \\
\hline ABCG2 & TATAGCTCAGATCATTGTCACAGTC & GTTGGTCGTCAGGAAGAAGAG \\
\hline $\mathrm{ABCC} 4$ & GGTTCCCCTTGGAATCATTT & AATCCTGGTGTGCATCAAACAG \\
\hline ABCC5 & ACCCGTTGTTGCCATCTTAG & GCTTTGACCCAGGCATACAT \\
\hline ERCC1 & CTCAAGGAGCTGGCTAAGATGT & CATAGGCCTTGTAGGTCTCCAG \\
\hline XRCC1 & CATCGTGCGTAAGGAGTGGGTG & AGTGGGCTTGGTTTTGGTCTGG \\
\hline MSH2 & GCTTCTCCTGGCAATCTCTCTC & TACCCAACTCCAACCTGTCTCT \\
\hline TUBB3 & TCAGCAAGGTGCGTGAGGAGTAT & CGGAAGCAGATGTCGTAGAGCG \\
\hline POLH & CGTGGGAGCAGTGATTGTGGAGGA & GGTTTGGCGGTTGGGCTTGTTTAG \\
\hline $\mathrm{GST} \pi$ & ACCTCCGCTGCAAATACATC & CTCAAAAGGCTTCAGTTGCC \\
\hline$\gamma \mathrm{GT} 1$ & GCCTGGATTCTCCCAGAGAT & GGAGAGCACCTCTTCCTCAG \\
\hline GAPDH & GCACCGTCAAGGCTGAGAAC & TGGTGAAGACGCCAGTGGA \\
\hline
\end{tabular}

each well. The plate was incubated at $37^{\circ} \mathrm{C}$ for $4 \mathrm{~h}$. Then, the culture medium was removed, and $100 \mathrm{ml}$ DMSO was added to each well to dissolve the dark blue crystal. The absorbance was measured at $570 \mathrm{~nm}$ using a microplate reader.

Immunohistochemistry. Sections cut from the human colorectal tumor paraffin blocks were subjected to deparaffinization/ rehydration, and antigen retrieval by boiling in $0.01 \mathrm{M}$ sodium citrate buffer for $30 \mathrm{~min}$. The sections were blocked with $10 \%$ goat serum, then incubated with the primary antibodies against Twist and P-gp at a dilution 1:200 at $4^{\circ} \mathrm{C}$ overnight in a humidified chamber. After washing with PBS three times, slides were incubated with HRP-conjugated antibody, and DAB was used as substrate. Mayer's hematoxylin was applied as a counter stain. Throughout the above analyses, controls were prepared by omitting the primary antibodies.

Statistical analysis. Results are expressed as mean \pm SD of three independent experiments unless otherwise specified. Data were analyzed by two-tailed unpaired Student's t-test between any two groups. One-way ANOVA analysis of variance was used to assess the difference of means among groups. These analyses were performed using GraphPad Prism Software Version 5.0 (GraphPad Software Inc., La Jolla, CA, USA). A P-value of $<0.05$ was considered statistically significant.

\section{Results}

Overexpression of Twist promotes migration and invasion of CRC cells. Tumor cells with an aggressive phenotype acquire invasive and migratory capabilities, which will promote the dissemination of tumors cells to distant organs (1). The migratory and invasive capabilities of HCT116 and SW480 cells transfected with vector plasmid or pcDNA-Twist were measured by using Transwell migration and invasion assays. As shown in Fig. 1A and B, overexpression of Twist led to an obvious increase in cell migration and invasion. Compared with control, the number of migrated and invasive cells increased about 5-fold (migration) and 15-fold (invasion) after overexpression of Twist.

The increased migratory and invasive capabilities of tumor cells are reminiscent of the molecular events at EMT. The morphology of HCT116 and SW480 cells was observed after transfection with pcDNA-Twist, we found that overexpression of Twist resulted in a significant change in cell morphology, from cobblestone phenotype to spindle-like and fusiform features (Fig. 1C).

Overexpression of Twist induces EMT of CRC cells. During EMT, the epithelial maker E-cadhein is downregulated, whereas the mesenchymal markers vimentin and $\mathrm{N}$-cadherin are upregulated (18). To confirm whether HCT116 and SW480 cells transfected with pcDNA-Twist truly acquire EMT features, western blotting was used to examine the expression of EMT makers. As shown in Fig. 2A, overexpression of Twist decreased E-cadherin expression, which is consistent with increased vimentin and N-cadherin. Similarly, real-time PCR analysis further confirmed the increasing expression of vimentin and $\mathrm{N}$-cadherin, and the decreased expression of E-cadherin at mRNA levels (Fig. 2B). Furthermore, immu- 
A

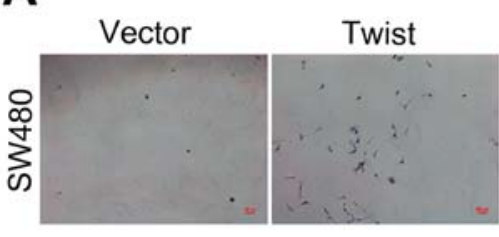

B
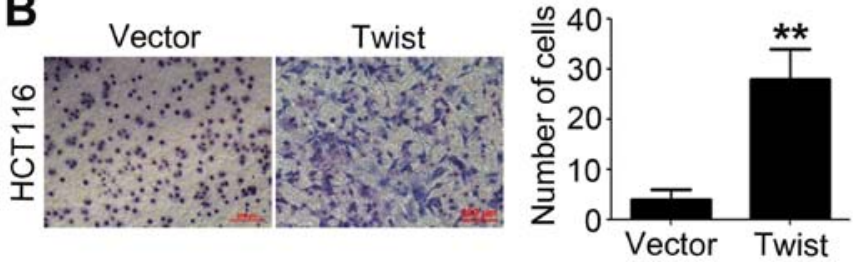

C



Figure 1. Overexpression of Twist promotes migration and invasion of CRC cells. (A) Left: SW480 cells transfected with pcDNA-3.1 (vector) or pcDNA-Twist (Twist) were allowed to migrate Transwell chambers for $24 \mathrm{~h}$. After $24 \mathrm{~h}$, the migrated cells were fixed, stained, and photographed. Magnification, x100. Right: The number of migrated cells. Data represent the average of three independent experiments. (B) Left: HCT116 cells transfected with pcDNA-3.1 or pcDNA-Twist were allowed to spread through the Matrigel and into the underside of the filter for $48 \mathrm{~h}$. Then the cells on the underside of the filter were fixed, stained, and photographed. Magnification, $x 200$. Right: The number of invasive cells. Data represent the average of three independent experiments. ${ }^{*} \mathrm{P}<0.05,{ }^{* *} \mathrm{P}<0.01$. (C) HCT116 and SW480 cells were transfected with pcDNA-3.1 or pcDNA-Twist. After $24 \mathrm{~h}$, cell morphological changes were shown in the phase contrast image.

nofluorescence analysis showed that overexpression of Twist downregulated E-cadherin and upregulated vimentin (Fig. 2C). Collectively, these observations suggested that HCT116 and SW480 cells had undergone EMT following overexpression of Twist.

Overexpression of Twist induces a CSC-like phenotype of CRC cells. To investigate whether cells with EMT phenotype could display CSC-like features, we detected mammosphere forming ability of HCT116 and SW480 cells transfected with pcDNA-Twist. We found that cells overexpressing Twist showed obviously enhanced ability to form mammosphere compared to control cells. As shown in Fig. 3A, overexpression of Twist increased the number of mammospheres about 3-7-fold of HCT116 and SW480 cells. To further investigate the genes maintaining and regulating the CSC properties, we performed western blotting and real-time PCR analysis. The results from western blotting showed that overexpression of
A

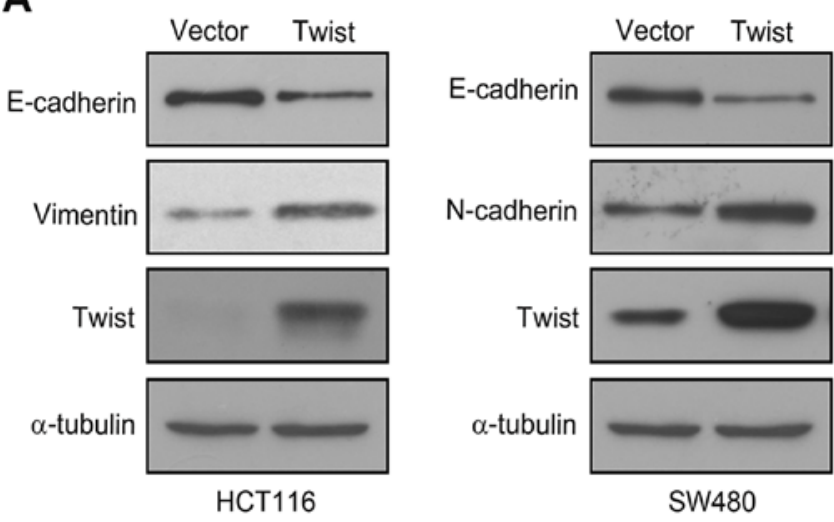

B
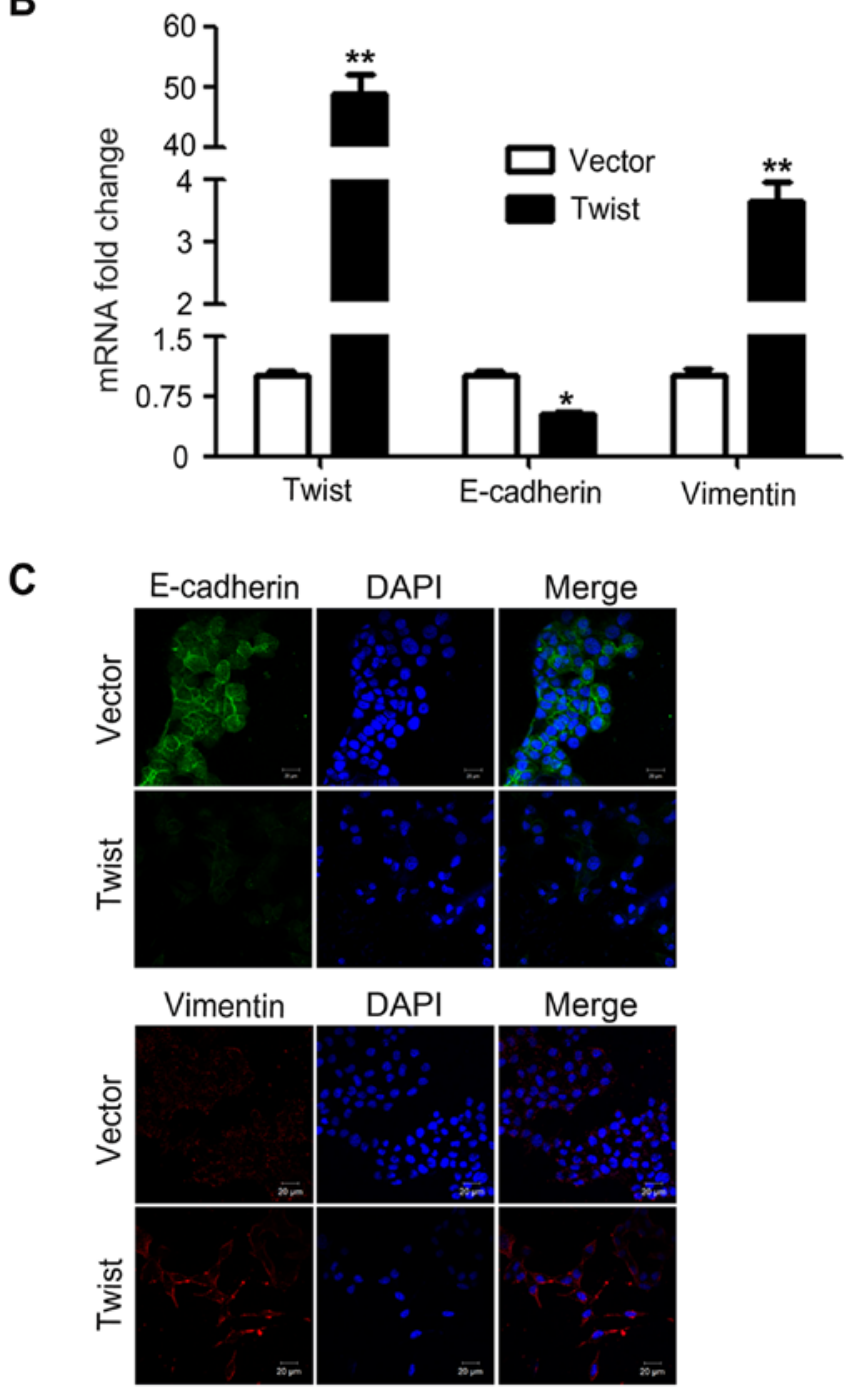

Figure 2. Overexpression of Twist induces EMT of CRC cells. (A) HCT116 and SW480 cells were transfected with pcDNA-3.1 or pcDNA-Twist for $48 \mathrm{~h}$, and the expression of E-cadherin, vimentin, $\mathrm{N}$-cadherin and Twist were analyzed by western blotting. $\alpha$-tubulin serves as the loading control. (B) HCT116 cells were transfected with pcDNA-3.1 or pcDNA-Twist for $24 \mathrm{~h}$. The mRNA levels of E-cadherin, vimentin and Twist were analyzed by qRT-PCR. "P $<0.05,{ }^{* *} \mathrm{P}<0.01$. (C) HCT116 cells were transfected with pcDNA-3.1 or pcDNA-Twist for $48 \mathrm{~h}$. Expression of E-cadherin and vimentin were analyzed by immunofluorescence staining. Nuclei were visualized with DAPI staining. Scale bars: $20 \mu \mathrm{m}$.

Twist significantly increased pluripotent makers Nanog and Bmi-1 expression levels (Fig. 3B). Real-time PCR analysis 
A HCT116




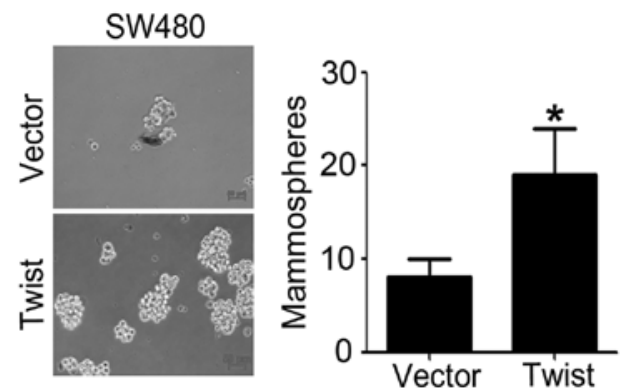

B
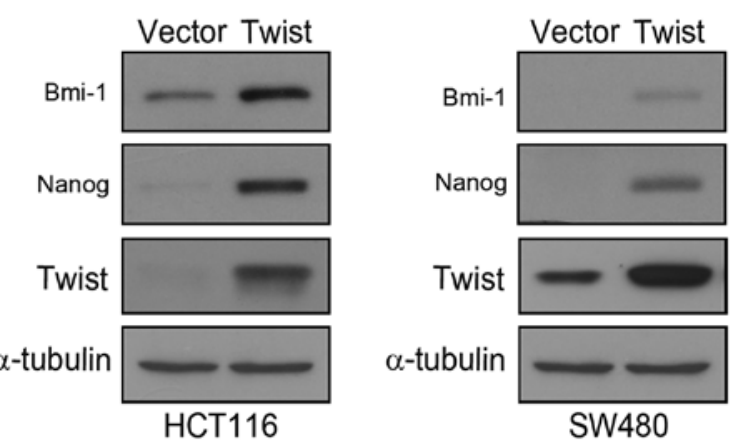

C

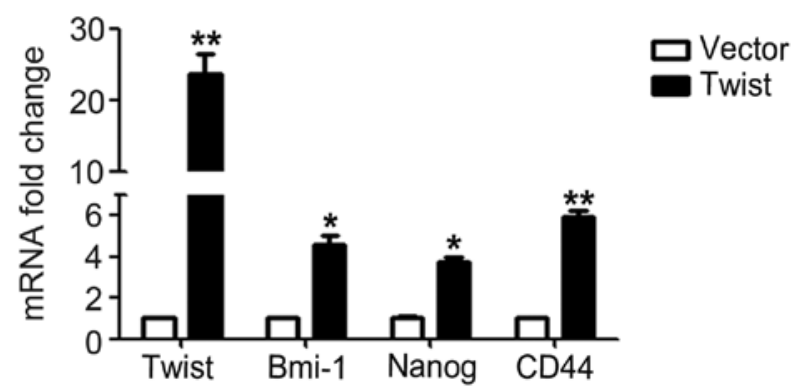

Figure 3. Overexpression of Twist induces a CSC-like phenotype of CRC cells. (A) pcDNA-3.1 or pcDNA-Twist was expressed in HCT116 and SW480 cells for $48 \mathrm{~h}$, mammosphere forming ability were examined. ${ }^{*} \mathrm{P}<0.05,{ }^{* *} \mathrm{P}<0.01$. (B) HCT116 and SW480 cells transfected with pcDNA-3.1 or pcDNA-Twist for $48 \mathrm{~h}$, the expression levels of Twist, Bmi-1 and Nanog were examined by western blotting. (C) Quantitative RT-PCR was used to quantify Twist, Bmi-1, Nanog and CD44 mRNA expression in HCT116 cells transfected with pcDNA-3.1 or pcDNA-Twist for $24 \mathrm{~h} .{ }^{*} \mathrm{P}<0.05,{ }^{* *} \mathrm{P}<0.01$.

also demonstrated that the mRNA expression levels of Nanog, Bmi-1 and CD44 are obviously enhanced in cells overexpressing Twist compared to control cells (Fig. 3C). These findings demonstrated that overexpression of Twist induces a CSC-like phenotype of CRC cells.

Overexpression of Twist mediates chemoresistance of CRC cells. Recent studies showed that EMT phenotype and CSC-like trait are closely related to chemoresistance of cancer cells (13). We next observed whether overexpression of Twist can affect sensitivity of HCT116 and SW480 cells to oxaliplatin. HCT116 and SW480 cells transfected with control vector plasmid or pcDNA-Twist were treated with different concentration of oxaliplatin and MTT assay was performed. The results showed that overexpression of Twist led to a distinct increase in cell viability compared with cells transfected with control vector (Fig. 4A). Underlying molecular mechanisms of chemoresistance are extremely complicated, involving ATP binding cassette (ABC) membrane transport proteins and DNA repair proteins (19). We overexpressed Twist and detected drug resistance-associated genes, including $\mathrm{ABCB} 1$, $\mathrm{ABCC} 1, \mathrm{ABCC} 2, \mathrm{ABCC} 3, \mathrm{ABCC} 4, \mathrm{ABCC} 5, \mathrm{ABCG} 2$,
ERCC1, XRCC1, MSH2, TUBB3, POLH, GST $\pi$ and $\gamma$-GT1. Noteworthy, overexpression of Twist distinctly increased the mRNA expression of ABCB1, but not the others (Fig. 4B). $\mathrm{P}-\mathrm{gp}$, a transmembrane glycoprotein characterized based on its ability to confer a multidrug-resistance phenotype to cancer cells is an ABCB1 encoded protein (20). Further, we demonstrated that overexpression of Twist also enhanced the expression of P-gp in HCT116 and SW480 cells (Fig. 4C). Taken together, these observations suggested that P-gp may be essential for drug resistant phenotype induced by Twist.

Clinical corelation of Twist and P-gp expression in human $C R C$ specimens. To further extend our findings in vivo, we determined whether there is a correlation between the expression of Twist and P-gp in resected human colorectal cancer specimens. Immunohistochemical analyses of Twist and P-gp were performed for 46 examples of tumor samples from colorectal cancer patients. A representative immunohistochemistry result is shown in Fig. 5, Twist expression was on the cell nuclei, while P-gp expressed on the cell membrane. Of note, expression of P-gp occurred in 28 cases $(60.8 \%)$ and expression of Twist occurred in 26 cases (56.5\%), indicating 

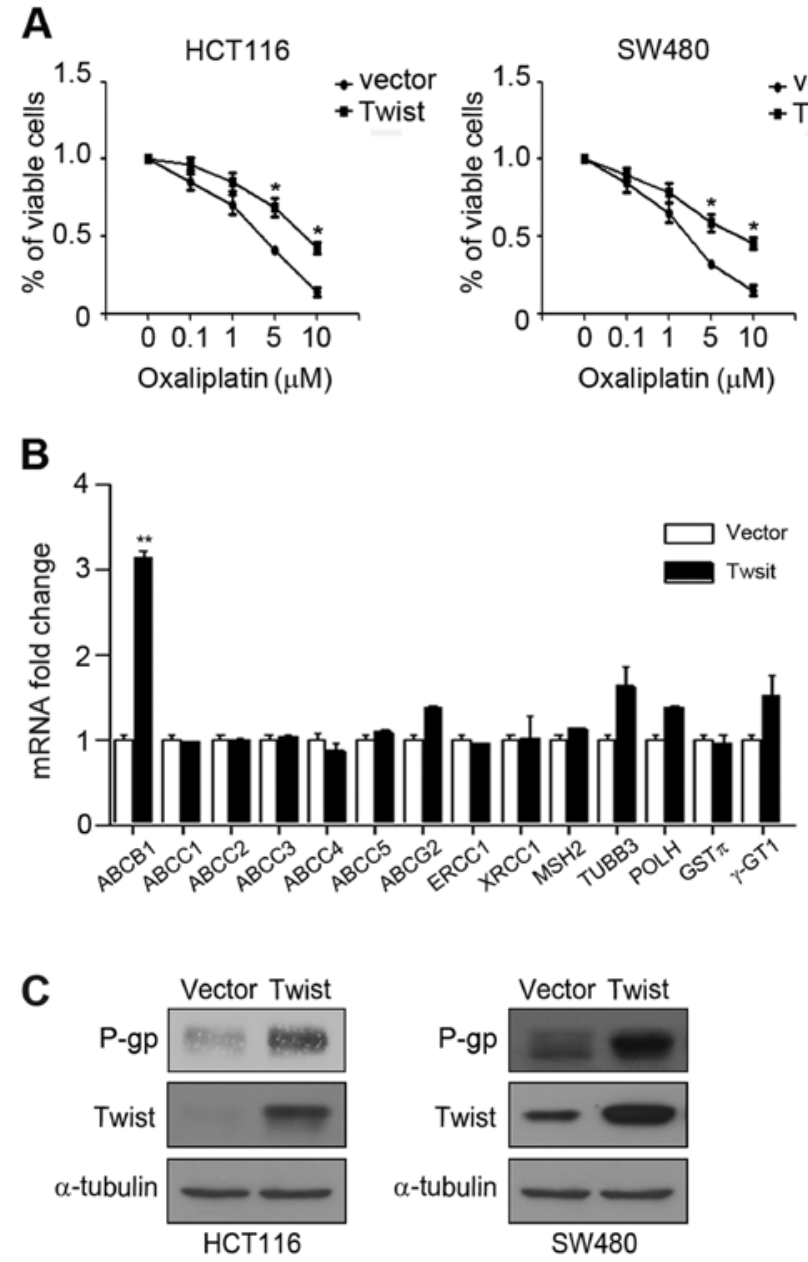

Figure 4. Overexpression of Twist mediates chemoresistance of CRC cells (A) HCT116 and SW480 cells were transfected with pcDNA-3.1 or pcDNATwist for $24 \mathrm{~h}$ and then treated with increasing concentrations of oxaliplatin for $48 \mathrm{~h}$. MTT assay was used to quantify the viable cells. "P<0.05. (B) Quantitative RT-PCR was used to quantify the drug resistance-associated gene mRNA expression in HCT116 cells transfected with pcDNA-3.1 or pcDNA-Twist for $24 \mathrm{~h} .{ }^{* *} \mathrm{P}<0.01$. (C) HCT116 and SW480 cells transfected with pcDNA-3.1 or pcDNA-Twist for $48 \mathrm{~h}$, the expression levels of Twist and P-gp were examined by western blotting.

that there was an obvious association between Twist and P-gp expression $(\mathrm{P}<0.01$; Table II).

\section{Discussion}

Metastasis involves many steps such as successful invasion, intravasation, survival in the circulation, extravasation and colonization by the cancer cells. Many cancer types depend on the process of EMT for successful completion of these steps (1). EMT is thought to be the first step of tumor invasion and metastasis (4). It has been reported that the expression profiles of EMT are associated with tumor grades and metastasis of breast carcinoma $(21,22)$. EMT also plays a crucial role in the metastasis of colorectal cancer, which occurs at the invasive front of colon carcinoma concomitant with a selective loss of basement membrane (23). Twist, a zinc-finger transcription factor, has been considered as a critical regulator that promotes EMT and induces invasiveness as well as metastasis in various types of malignant tumors (24). In CRC
Table II. Immunohistochemical staining of Twist and P-gp was performed in 46 tumor samples from colorectal cancer patients. $^{\mathrm{a}}$

\begin{tabular}{lrcrr}
\hline & \multicolumn{3}{c}{ P-gp } & \\
\cline { 2 - 3 } & Positive & Negative & Total & P-value \\
\hline Twist & & & & \\
Positive & 21 & 5 & 26 & \\
Negative & 7 & 13 & 20 & $<0.01$ \\
Total & 28 & 18 & 46 & \\
\hline
\end{tabular}

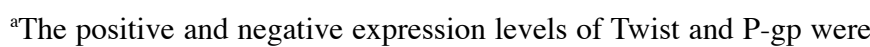
counted and analyzed. $\mathrm{P}<0.01$.

tissues, Twist was highly expressed and the inverse correlation between Twist and E-cadherin was observed (25). In addition, aberrant Twist expression associated significantly with lymph node metastasis of CRC (25). Consistent with the above investigations, our studies demonstrated that overexpression of Twist in CRC cells led to decreased E-cadherin expression and increased vimentin and $\mathrm{N}$-cadherin expression. Furthermore, acquisition of mesenchymal phenotype has been associated with enhanced invasive and metastatic behavior.

Recently, it was proposed that EMT not only enables cancer cells to disseminate but also to acquire the ability to self-renew by inducing a CSC trait (26). The CSCs represent a small subset of cells within a malignant tumor thought to be capable of initiating the tumor and of driving its growth and recurrence after treatment (14). The relationship between EMT and CSC has been the focus of recent research. Mani et al demonstrated that overexpression of EMT regulators Snail and Twist in immortalized human mammary epithelial cells (HMLEs) induced an EMT state and conferred to the cells an increased ability to form mammospheres, which is characteristic of CSCs (27). Comparably, McCoy et al proved that the homeobox transcription factor Six 1 induced tumors that underwent EMT in mouse mammary gland epithelium by increasing the population of cells displaying CSC markers (28). Similarly, recent research showed that gene expression patterns of CSC-associated pathways were involved in EMT (29). In line with these studies, we found that Twist-induced EMT conferred a CSC-like phenotype in CRC cells. Overexpression of Twist in two CRC cell lines resulted in increased expression of the CSC markers Bmi-1, Nanog and CD44 as well as enhanced ability to form mammospheres.

Increasing evidence suggested that both EMT and CSC phenotype are associated with chemoresistance (13). Cancer cells undergoing EMT become resistant to chemotherapy, and cancer cells selected for chemoresistance acquire EMT phenotype $(1,16,30,31)$. Induction of EMT was detected to promote the decreased efficacy of chemotherapy in colorectal, breast and ovarian cancers $(30,32,33)$. It has been demonstrated that chemoresistance is due to the existence of CSC features, which remain after treatment and result in cancer relapse. In this study, we also showed that the CRC cells overexpressing Twist exhibited EMT and CSC properties as well as resistance 


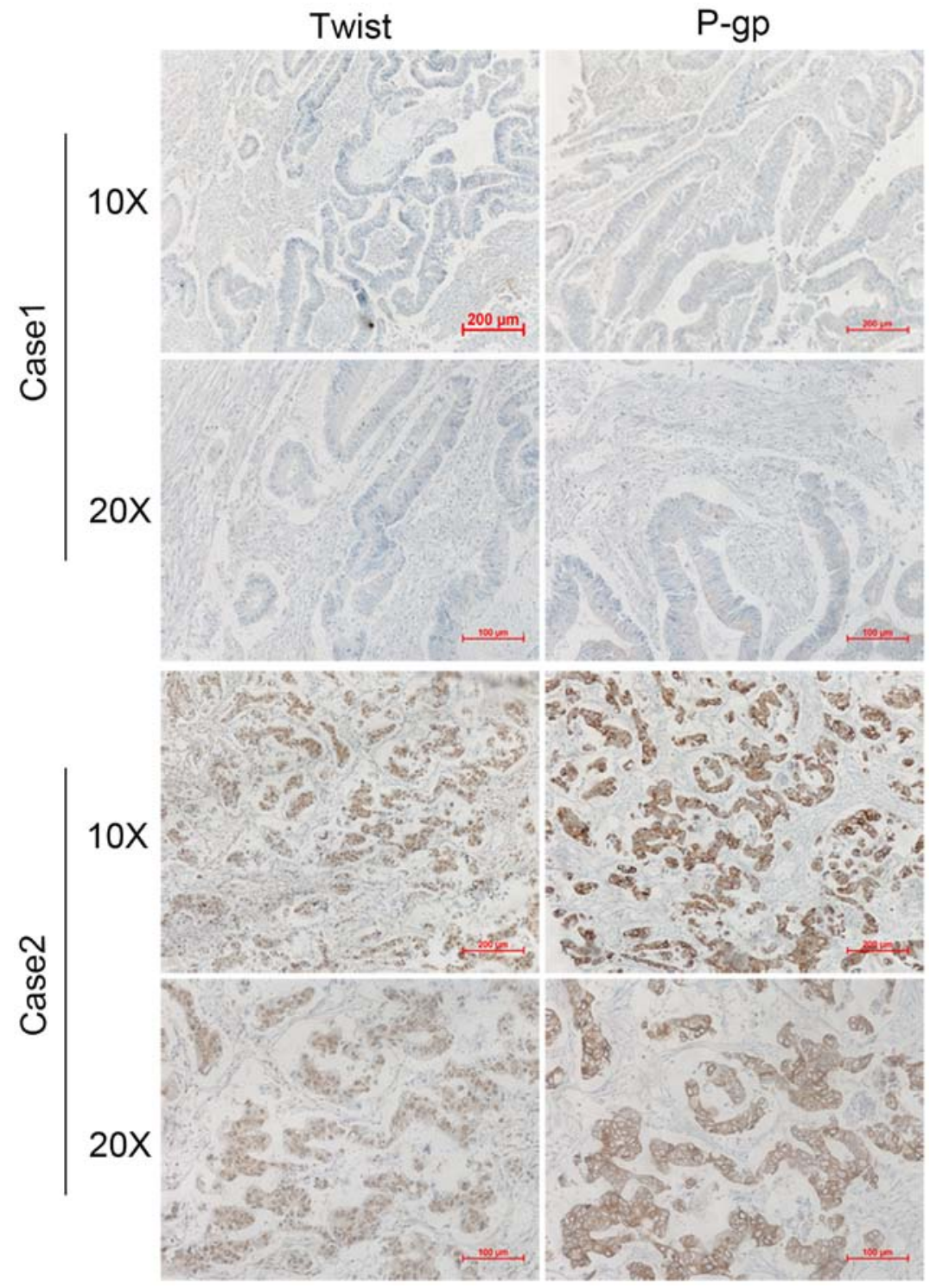

Figure 5. Twist and P-gp are correlatively expressed in human CRC specimens. Immunohistochemical staining of Twist and P-gp in two representative colorectal tumor cases which were negative for Twist and P-gp expression (Case 1) and co-expression of Twist and P-gp (Case 2). Scale bars, 100 or $200 \mu \mathrm{m}$.

to chemotherapy. The cancer cells resistant to conventional therapies was primary due to altered expression of ATP binding cassette (ABC) membrane transport proteins (34), overexpression of the DNA repair enzymes (35), activation of DNA damage checkpoint responses and inhibition of cell apoptosis $(19,36)$.

We next investigated the underlying mechanism of chemoresistance induced by Twist overexpression and our results showed that Twist significantly enhance ABCB1 mRNA level, but not the other drug resistance genes, such as $\mathrm{ABCC} 1, \mathrm{ABCC} 2, \mathrm{ABCC} 3, \mathrm{ABCC} 4, \mathrm{ABCC} 5, \mathrm{ABCG} 2$, ERCC1, XRCC1, MSH2, TUBB3, POLH, GST $\pi$ and $\gamma$-GT1. A recent study found that Twist can directly combine ABCC4 and $\mathrm{ABCC} 5$ promoters and increase their expression levels in breast epithelial cells (37). However, in our study, we did not find this phenomenon, which may be due to the different cell lines. P-gp, a transmembrane glycoprotein encoded by the ABCB1 gene, is the first and most important human ABC transporter and confer a multidrug-resistance phenotype to cancer cells (20). Clinically, P-gp is involved in the process of local metastatic dissemination and drug resistance of colorectal carcinoma cells (38). We further confirmed that high expression of Twist increased P-gp expression in CRC cells. Importantly, we found clinical correlation of Twist and P-gp expression in human CRC specimens. Software analysis showed that the promoter of $\mathrm{ABCB} 1$ have Twist binding sites (CANNTG). It is worth further investigation to assess whether Twist can directly combine the promoter of ABCB1 and promote P-gp expression.

In summary, our studies have demonstrated that Twist expression in human CRC cells can mediate EMT and the development of the CSC phenotype as well as chemoresistance. Therapeutic targeting of Twist may be a novel method to enhance the efficacy of chemotherapy and improve outcomes for patients with metastatic CRC.

\section{Acknowledgements}

This work was funded by the Natural Science Foundation of Hubei Province (no. 2015CFB206). 


\section{References}

1. Thiery JP, Acloque H, Huang RY and Nieto MA: Epithelialmesenchymal transitions in development and disease. Cell 139: 871-890, 2009

2. Kalluri R and Weinberg RA: The basics of epithelial-mesenchymal transition. J Clin Invest 119: 1420-1428, 2009.

3. Wu Y and Zhou BP: Snail: More than EMT. Cell Adh Migr 4 199-203, 2010

4. Christofori G: New signals from the invasive front. Nature 441: 444-450, 2006.

5. Barrallo-Gimeno A and Nieto MA: The Snail genes as inducers of cell movement and survival: implications in development and cancer. Development 132: 3151-3161, 2005.

6. Wang H, Wang HS, Zhou BH, Li CL, Zhang F, Wang XF, Zhang G, Bu XZ, Cai SH and Du J: Epithelial-mesenchymal transition (EMT) induced by TNF- $\alpha$ requires AKT/GSK-3 $\beta$ mediated stabilization of snail in colorectal cancer. PLoS One 8: e56664, 2013.

7. Thiery JP and Sleeman JP: Complex networks orchestrate epithelial-mesenchymal transitions. Nat Rev Mol Cell Biol 7: $131-142,2006$

8. Peinado H, Olmeda D and Cano A: Snail, Zeb and bHLH factors in tumour progression: an alliance against the epithelial phenotype? Nat Rev Cancer 7: 415-428, 2007.

9. Nuti SV, Mor G, Li P and Yin G: TWIST and ovarian cancer stem cells: implications for chemoresistance and metastasis. Oncotarget 5: 7260-7271, 2014.

10. Wushou A, Hou J, Zhao YJ and Shao ZM: Twist-1 up-regulation in carcinoma correlates to poor survival. Int J Mol Sci 15 21621-21630, 2014

11. Kwok WK, Ling MT, Lee TW, Lau TC, Zhou C, Zhang X, Chua CW, Chan KW, Chan FL, Glackin C, et al: Up-regulation of TWIST in prostate cancer and its implication as a therapeutic target. Cancer Res 65: 5153-5162, 2005.

12. Zhang Z, Xie D, Li X, Wong YC, Xin D, Guan XY, Chua CW, Leung SC, Na Y and Wang X: Significance of TWIST expression and its association with E-cadherin in bladder cancer. Hum Pathol 38: 598-606, 2007.

13. Singh A and Settleman J: EMT, cancer stem cells and drug resistance: an emerging axis of evil in the war on cancer. Oncogene 29: 4741-4751, 2010.

14. Kong D, Li Y, Wang Z and Sarkar FH: Cancer stem cells and epithelial-to-mesenchymal transition (EMT)-phenotypic cells: are they cousins or twins? Cancers (Basel) 3: 716-729, 2011

15. Kong D, Banerjee S, Ahmad A, Li Y, Wang Z, Sethi S and Sarkar FH: Epithelial to mesenchymal transition is mechanistically linked with stem cell signatures in prostate cancer cells. PLoS One 5: e12445, 2010.

16. Wang H, Zhang G, Zhang H, Zhang F, Zhou B, Ning F, Wang HS, Cai SH and Du J: Acquisition of epithelial-mesenchymal transition phenotype and cancer stem cell-like properties in cisplatin-resistant lung cancer cells through AKT/ $\beta$-catenin/ Snail signaling pathway. Eur J Pharmacol 723: 156-166, 2014.

17. Kim YH, Kim G, Kwon CI, Kim JW, Park PW and Hahm KB: TWIST1 and SNAI1 as markers of poor prognosis in human colorectal cancer are associated with the expression of ALDH1 and TGF- $\beta 1$. Oncol Rep 31: 1380-1388, 2014.

18. Voulgari A and Pintzas A: Epithelial-mesenchymal transition in cancer metastasis: mechanisms, markers and strategies to overcome drug resistance in the clinic. Biochim Biophys Acta 1796: 75-90, 2009.

19. He H, Ni J and Huang J: Molecular mechanisms of chemoresistance in osteosarcoma (Review). Oncol Lett 7: 1352-1362, 2014.

20. Xue $X$ and Liang XJ: Overcoming drug efflux-based multidrug resistance in cancer with nanotechnology. Chin J Cancer 31: 100-109, 2012

21. Logullo AF, Nonogaki S, Pasini FS, Osório CA, Soares FA and Brentani MM: Concomitant expression of epithelialmesenchymal transition biomarkers in breast ductal carcinoma: Association with progression. Oncol Rep 23: 313-320, 2010.
22. Xue CS, Plieth D, Venkov C, Xu C, Neilson EG: The gatekeeper effect of epithelial-mesenchymal transition regulates the frequency of breast cancer metastasis. Cancer Res 63: 3386-3394, 2003.

23. Sheehan KM, Gulmann C, Eichler GS, Weinstein JN, Barrett HL, Kay EW, Conroy RM, Liotta LA and Petricoin EF III: Signal pathway profiling of epithelial and stromal compartments of colonic carcinoma reveals epithelial-mesenchymal transition. Oncogene 27: 323-331, 2008

24. Jung HY and Yang J: Unraveling the TWIST between EMT and cancer stemness. Cell Stem Cell 16: 1-2, 2015.

25. Galván JA, Helbling M, Koelzer VH, Tschan MP, Berger MD, Hädrich M, Schnüriger B, Karamitopoulou E, Dawson H, Inderbitzin D, et al: TWIST1 and TWIST2 promoter methylation and protein expression in tumor stroma influence the epithelialmesenchymal transition-like tumor budding phenotype in colorectal cancer. Oncotarget 6: 874-885, 2015.

26. Fan YL, Zheng M, Tang YL and Liang XH: A new perspective of vasculogenic mimicry: EMT and cancer stem cells (Review). Oncol Lett 6: 1174-1180, 2013.

27. Mani SA, Guo W, Liao MJ, Eaton EN, Ayyanan A, Zhou AY, Brooks M, Reinhard F, Zhang CC, Shipitsin M, et al: The epithelial-mesenchymal transition generates cells with properties of stem cells. Cell 133: 704-715, 2008.

28. McCoy EL, Iwanaga R, Jedlicka P, Abbey NS, Chodosh LA, Heichman KA, Welm AL and Ford HL: Six1 expands the mouse mammary epithelial stem/progenitor cell pool and induces mammary tumors that undergo epithelial-mesenchymal transition. J Clin Invest 119: 2663-2677, 2009.

29. Gupta PB, Onder TT, Jiang G, Tao K, Kuperwasser C, Weinberg RA and Lander ES: Identification of selective inhibitors of cancer stem cells by high-throughput screening. Cell 138: 645-659, 2009.

30. Yang AD, Fan F, Camp ER, van Buren G, Liu W, Somcio R, Gray MJ, Cheng H, Hoff PM and Ellis LM: Chronic oxaliplatin resistance induces epithelial-to-mesenchymal transition in colorectal cancer cell lines. Clin Cancer Res 12: 4147-4153, 2006.

31. Arumugam T, Ramachandran V, Fournier KF, Wang H, Marquis L, Abbruzzese JL, Gallick GE, Logsdon CD, McConkey DJ and Choi W: Epithelial to mesenchymal transition contributes to drug resistance in pancreatic cancer. Cancer Res 69: 5820-5828, 2009.

32. Cheng GZ, Chan J, Wang Q, Zhang W, Sun CD and Wang LH: Twist transcriptionally up-regulates AKT2 in breast cancer cells leading to increased migration, invasion, and resistance to paclitaxel. Cancer Res 67: 1979-1987, 2007.

33. Kajiyama H, Shibata K, Terauchi M, Yamashita M, Ino K, Nawa A and Kikkawa F: Chemoresistance to paclitaxel induces epithelial-mesenchymal transition and enhances metastatic potential for epithelial ovarian carcinoma cells. Int J Oncol 31: 277-283, 2007

34. Fukuda Y and Schuetz JD: ABC transporters and their role in nucleoside and nucleotide drug resistance. Biochem Pharmacol 83: 1073-1083, 2012

35. Hsu DS, Lan HY, Huang CH, Tai SK, Chang SY, Tsai TL, Chang CC, Tzeng $\mathrm{CH}, \mathrm{Wu} \mathrm{KJ}$, Kao JY, et al. Regulation of excision repair cross-complementation group 1 by Snail contributes to cisplatin resistance in head and neck cancer. Clin Cancer Res 16: 4561-4571, 2010.

36. Bao S, Wu Q, McLendon RE, Hao Y, Shi Q, Hjelmeland AB, Dewhirst MW, Bigner DD and Rich JN: Glioma stem cells promote radioresistance by preferential activation of the DNA damage response. Nature 444: 756-760, 2006.

37. Saxena M, Stephens MA, Pathak H and Rangarajan A: Transcription factors that mediate epithelial-mesenchymal transition lead to multidrug resistance by upregulating $\mathrm{ABC}$ transporterss. Cell Death Dis 2: e179, 2011.

38. Zorzos HS, Lazaris AC, Korkolopoulou PA, Kavantzas NG, Tseleni-Balafouta S, Patsouris ES, Tsavaris NV and Davaris PS: Multidrug resistance proteins and topoisomerase IIalpha expression in colon cancer: association with metastatic potential. Pathology 35: 315-318, 2003. 